\title{
LncRNA Hoxb3os protects podocytes from high glucose-induced cell injury through autophagy dependent on the Akt-mTOR signaling pathway
}

\author{
Juan Jin, Jianguang Gong, Li Zhao, Yiwen Li and Qiang He区 \\ Nephrology Center, Department of Nephrology, Zhejiang Provincial People's Hospital, Affiliated People's Hospital, Hangzhou Medical College, \\ Hangzhou, Zhejiang, China
}

Background: Diabetic nephropathy (DN) is in the first place of the causes that lead to end-stage renal disease in the world. Thus, it is urgent to develop a novel diagnostic or therapeutic strategy that could stop the progression of diabetic nephropathy. Methods: RNA-sequencing was conducted in high glucose (HG)-treated MPC5 cells (podocytes). Cell morphology was examined under a light microscope. Upon high-glucose challenge, the effects of IncRNA Hoxb3os overexpression on MPC5 cells apoptosis, viability, autophagy and Akt-mTOR signaling were evaluated using flow cytometry, Cell Counting Kit-8, qRT-PCR, and Western blotting. TUNEL staining and ELISA were performed to confirm the establishment of DN model in $\mathrm{db} / \mathrm{db}$ mice. Results: High-glucose exposure dramatically altered IncRNA expression profile in MPC5 cells (fold change $>2$ ), including 305 upregulated IncRNAs and 451 downregulated IncRNAs. LncRNA Hoxb3os expression was significantly reduced in the HG-induced podocyte damage model, as well as in the renal tissues from $\mathrm{db} / \mathrm{db}$ mice with spontaneous $\mathrm{DN}$. Overexpression of Hoxb3os significantly reduced the apoptosis rate and increased the viability of MPC5 cells under HG conditions. Further study revealed that exogenous Hoxb3os increased autophagy level in HG-exposed MPC5 cells via abrogating Akt-mTOR signaling pathway and that the process was possibly implicated in the upregulation of SIRT1. Conclusion: LncRNA Hoxb3os protected podocytes from HG-induced damage by regulating Akt-mTOR pathway and cell autophagy. Thus, IncRNA Hoxb3os appears as a potential biomarker in the diagnosis and treatment of DN in the future.

Keywords: diabetic nephropathy, Hoxb3os, podocyte, high glucose, autophagy

Received: 08 September, 2020; revised: 04 November, 2020; accepted: 09 December, 2020; available on-line: 14 October, 2021

⿶e-mail: hegiangzj@outlook.com

Acknowledgements of Financial Support: This study was funded by the Provincial Foundation Project (Grant number WKJ-ZJ-1915). Abbreviations: DN, diabetic nephropathy; HG, high glucose; IncRNAs, Long noncoding RNAs; PVDF, polyvinylidene fluoride

\section{INTRODUCTION}

Diabetic nephropathy (DN), a serious advanced complication of diabetes with few effective treatments and characterized by glomerular hypertrophy, albuminuria, and accumulation of glomerular matrix, ultimately culminating in tubulointerstitial fibrosis, is the leading global cause of end-stage renal disease (Gaede et al., 2008; Haneda et al., 2015; Marshall, 2012; Rosolowsky et al., 2011). Therefore, it is crucial to develop a novel diagnostic or therapeutic strategy against renal injury in order to improve the prognosis of patients with DN. Podocytes are predominantly responsible for maintaining the glomerular filtration barrier, whose injuries facilitate the progression of DN. Unfortunately, the molecular mechanisms involved in the podocytes injury remain largely unclear. limited.

Long noncoding RNAs (lncRNAs) are a heterogeneous class of long (>200 nucleotides) transcripts with an apparent lack of protein-coding potential ${ }^{5}$. Increasing evidence has indicated that lncRNAs play crucial roles in the development of DN. For instance, lncRNA TUG1 in renal tissue exerts a protective role against $\mathrm{DN}$ by alleviating podocyte damage via modulating mitochondria function and metabolism (Li \& Susztak, 2016; Long et al., 2016). Re-expression of lncRNA 01619 in DN patients prevents podocyte damage and DN development (Bai et al., 2018). LncRNA 1700020 I14 Rik alleviates cell proliferation and fibrosis in mouse mesangial cells via miR-34a-5p/Sirt1/HIF-1 $\alpha$ signaling, slowing down DN progression (Li et al., 2018).

It was reported that high-glucose $(\mathrm{HG})$ challenge leads to dramatically reduced autophagy activity in podocytes cultured in vitro (Liu et al., 2016b). Autophagy inhibition results in the enhanced podocyte damage and excessive proteinuria, which contribute to DN progression (Liu et al., 2016a). Our previous studies also demonstrated that autophagy activation mitigates podocyte damage and thus inhibits DN pathogenesis (Jin et al., 2019a; Jin et al., 2019b), suggesting the crucial role of cellular autophagy in preventing podocyte damage. Of note, it was reported that IncRNA Hoxb3os deficiency promotes the phosphorylation of a pivotal autophagy regulatormTOR (Aboudehen et al., 2018), indicating the potential role of lncRNA Hoxb3os in modulating autophagy. In the present study, we identified a novel lncRNA - Hoxb3os - in podocytes. HG-induced podocyte injury model was established. We investigated the expression level of Hoxb3os in podocytes under HG treatment and kidney tissue from $\mathrm{db} / \mathrm{db}$ mice as $\mathrm{DN}$ model. The role of Hoxb3os in protecting the podocytes from HG-induced cell viability decrease and apoptosis promotion was revealed. Furthermore, we evaluated the effects of Hoxb3os on autophagy level, SIRT1 expression and Akt-mTOR signaling pathway in podocytes treated with HG.

\section{METHODS AND MATERIALS}

\section{Cell culture and treatment}

MPC5 podocyte cell line was obtained from Center for Kidney Disease, Second Affiliated Hospital of Nan- 
jing Medical University and were cultured in RPMI 1640 medium supplemented with $10 \%$ fetal bovine serum (Invitrogen, NY, USA) at $33^{\circ} \mathrm{C}$ in a humidified atmosphere of $5 \% \mathrm{CO}_{2}$. After culturing to a confluence of $80-90 \%$, the podocytes were sub-cultured under similar conditions for 10-14 days to induce cell differentiation at $37^{\circ} \mathrm{C}$. For the $\mathrm{HG}$ model establishment, the cells were exposed to the high-glucose (HG, $30 \mathrm{mM} \mathrm{D}$ glucose) or normal-glucose (NG, $5 \mathrm{mM} \mathrm{D}$-glucose) as the negative control. For inhibitor treatment, HG-exposed MPC5 cells or HG-exposed and Hoxb3os-overexpressing MPC5 cells were treated with transcription inhibitor actinomycin D (ACTD, 500 nM, HY-13918, MCE, USA) or translation inhibitor cycloheximide (CHX, $40 \mu \mathrm{g} / \mathrm{ml}, 40325 E S 03$, YEASEN, Shanghai, $\mathrm{CHN}$ ) for $24 \mathrm{~h}$. Subsequently, the cells were harvested for the experiments.

\section{Transcriptome sequencing}

Total RNA was extracted using RNeasy Mini Kit (Qiagen, Germany) and the concentration, integrity and RNA integrity number (RIN) parameters were assessed using Agilent 2100 Bioanalyzer (Agilent RNA 6000 Nano Kit). RNA preparation, library construction and sequencing were performed using the Illumina HiSeq 4000 platform (Illumina, San Diego, CA, USA) at the Beijing Genomics Institute (BGI, Shenzhen, China). Moderated $\log _{2}$-fold change values and BenjaminiHochberg-corrected $p$ values were calculated using DESeq2 (1.12. 3, R packges, Revolution Analytics, Microsoft). The statistical analysis was performed, and differentially expressed genes (DEGs) were selected that met the criteria of a fold change $>2, P<0.05$ and false discovery rate $(\mathrm{FDR})<0.05$.

\section{Microscopy}

MPC5 cells were cultured under high-glucose or normal-glucose conditions for $72 \mathrm{~h}$, and cell morphology and apoptotic cells in glomeruli of DN mice of apoptotic cells in glomeruli were examined under a light microscope.

\section{CCK-8 assay}

After treatment with high glucose or normal glucose or infection with lentivirus, the viability of MPC5 cells was analyzed using CCK-8 assay. $20 \mu \mathrm{L}$ cell counting solution (Beyotime, C0037) was added to the culture medium and incubated in the dark at $37^{\circ} \mathrm{C}$ for $4 \mathrm{~h}$. The absorption at $450 \mathrm{~nm}$ was evaluated using a microplate reader (Bio-Rad, Hercules, California, US).

\section{Flow cytometry}

The cultured MPC5 cells were digested with trypsin, washed with cold PBS and double-stained with AnnexinV-FITC/propidium iodide (C1062M, Beyotime, Shanghai, CHN) according to the manufacturer's instructions. Apoptosis was detected by flow cytometry using BD FACSCalibur (Becton, NJ, USA).

\section{Quantitative real-time PCR}

Total RNA was extracted using TRIzol Reagent (Invitrogen, CA, USA) according to the manufacturer's instruction. $1 \mu \mathrm{g}$ of total RNA was reversely transcribed to cDNA using an RNA PCR Kit (Takara, Japan). To detect gene expression, quantitative real-time PCR (qRTPCR) was performed using an iCycler iQ System with the iQ SYBR Green Super Mix (BioRad, USA) according to the manufacturer's instructions. GAPDH was used as an internal control for the normalization of expression level. The relative gene expression level was calculated using $\left(2^{-\Delta \Delta C t}\right)$ method.

\section{Northern blot}

$20 \mu \mathrm{g}$ RNA was resolved in $12 \%$ denaturing NovexTM TBE-Urea Gel (Invitrogen, CA, USA) and transferred to a Hybond-N+ nylon membrane in a semi-dry electroblotter. Subsequently, UV crosslinking was carried out by exposure to the ultraviolet lamp $\left(1,500 \mathrm{~mJ} / \mathrm{cm}^{2}\right)$ for $1 \mathrm{~min}$. The probe for Hoxb3os (DIG-AGCAGAAGGTTGTGGTGGT) was then incubated with the membranes in hybridization buffer $(10 \%$ SDS, $10 \%$ dextran sulfate, $1 \mathrm{M} \mathrm{NaCl}, 0.5 \mathrm{mg} / \mathrm{mL}$ sonicated salmon sperm DNA) at $65^{\circ} \mathrm{C}$ overnight. Then, the membranes were incubated in $0.3 \times$ SSC and $1 \%$ SDS at $65^{\circ} \mathrm{C}$ for $1 \mathrm{~h}$. Finally, the blots were visualized using a ChemiDoc MP system (Biorad, USA).

\section{DN model establishment}

10-week-old female C57BL/Ks db/db mice (DN model) and $\mathrm{C} 57 \mathrm{BL} / \mathrm{KsJ} \mathrm{db} / \mathrm{m}$ mice (normal mice) were obtained from Vital River company (Beijing, China). Animal experiments were approved by the ethics committee of Zhejiang Provincial People's Hospital and were performed in accordance with the standards of the NIH Instructions for the Care and Use of Laboratory Animals. Mice were housed in the animal facility at $23 \pm 3^{\circ} \mathrm{C}$ under a $12 \mathrm{~h}$ light/dark cycle. After 15 weeks, the kidney cortex, serum and urine were obtained for further analysis.

\section{Terminal Deoxynucleotide Transferase dUTP Nick End Labeling (TUNEL) assay}

TUNEL Assay was performed using an appropriate kit (Roche Applied Science, Mannheim, Germany). The kidney tissues were rinsed, dehydrated, embedded in paraffin and cut into $4-\mu \mathrm{m}$-thick slices. The experimental procedures were in accordance with the manufacturer's instructions. Slices were mounted and the number of TUNEL-positive nuclei was calculated, then the percentage of apoptotic cells in glomerulus was calculated.

\section{Assessments of Biochemical Parameters}

The levels of BUN and Scr (Jiancheng Bioengineering Institute, Nanjing, China) in the serum of mice were detected using specific kits according to the manufacturer's instructions. The urinary proteins were extracted using a Liquid Protein Extraction kit- II (Applygen Technologies, Beijing, China), then the concentration of urine protein was measured using a BCA kit (P1511, Applygen Technologies Inc., Beijing, China).

\section{Overexpression of Hoxb3os}

Full-length Hoxb3os (1,945 bp) was amplified using primers as follows: reverse, 5'-tcacgtgacagcctcattg-3' and forward, 5'-aagagattccagcagatgca-3', followed by cloning into a lentiviral vector containing a modified backbone (LeGO-CeB/lnc vector) (Emmrich et al., 2014). Subsequently, MPC5 cells were infected with Hoxb3os overexpression lentivirus (HanBio, Shanghai, China) at MOI $=10$ in the presence of $10 \mu \mathrm{g} / \mathrm{ml}$ polybrene. Four hours after infection, the supernatant was replaced with a fresh medium. Cells were cultured for 72 hours then harvested for further experiments. 


\section{Western blot}

The protein samples were extracted from the podocyte cells using a lysis buffer. $40 \mu \mathrm{g}$ protein was separated in 10\% sodium dodecyl sulfate-polyacrylamide gel (SDS-PAGE) and transferred to polyvinylidene fluoride (PVDF) membranes. The blots were incubated with $5 \%$ skimmed milk in PBS for $2 \mathrm{~h}$ to block non-specific binding followed by incubation with primary antibodies against LC3 (Abcam, ab48394, 1:1,000, UK), p62 (Abcam, ab109012, 1:1,000, UK), Akt (CST, 9272, 1:1,000, USA), p-Akt (CST, 4060, 1:1,000, USA), mTOR (CST, 2972, 1:1,000, USA), p-mTOR (CST, 5536, 1:1,000, USA), SIRT1 (Abcam, ab189494, 1:1,000, UK) and GAPDH (Abcam, ab181602, 1:1,000, UK), overnight at $4^{\circ} \mathrm{C}$. After 3 washes with PBST, the blots were incubated with horseradish peroxidase-conjugated goat anti-rabbit antibodies at room temperature for $90 \mathrm{~min}$. GAPDH was used as an internal control. Finally, the blots were treated with ECL plus reagent (Pierce, IL, USA) and visualized using charged-coupled device LAS 4000 (Fujifilm, Valhalla, NY, USA).

\section{Statistical Analysis}

All the data are presented as the means \pm S.D. Oneway ANOVA was used to assess the differences between multiple groups. Differences between two groups were analyzed using the Student's $t$-test. $P<0.05$ was considered statistically significant.

\section{RESULTS}

\section{High-glucose exposure alters IncRNA expression profile}

Firstly, in order to investigate the roles of lncRNAs in podocytes upon HG challenge, we performed RNA-Seq using cells under normal-glucose and HG treatment. A total of $756 \operatorname{lncRNAs}$ were found to be prominently altered (fold change $>2$ ) by high-glucose exposure (Fig. 1A-B). These differentially-expressed lncRNAs contained 305 upregulated lncRNAs and 451 downregulated lncRNAs. There were 163 lncRNAs in total that displayed more than 5-fold change, including 54 upregulated lncRNAs and 109 downregulated lncRNAs, and a total of 68 lncRNAs displayed more than 10-fold change, including 23 upregulated lncRNAs and 45 downregulated lncRNAs (Fig. 1B). We then analyzed the top 5 upregulated as well as the top 5 downregulated lncRNAs (Fig. 1C) and found a dramatically down-regulated lncRNA Hoxb3os (ENSMUST00000147410), which was also reported to be lowered in the renal tissues obtained from autosomal dominant polycystic kidney disease (ADPDK) mice ${ }^{12}$. The level of its human counterpart, lncRNA HOXBAS1, is also reduced in renal samples from ADPDK patients (Bai et al., 2018). Therefore, lncRNA Hoxb3os was selected for the subsequent experiments.
A

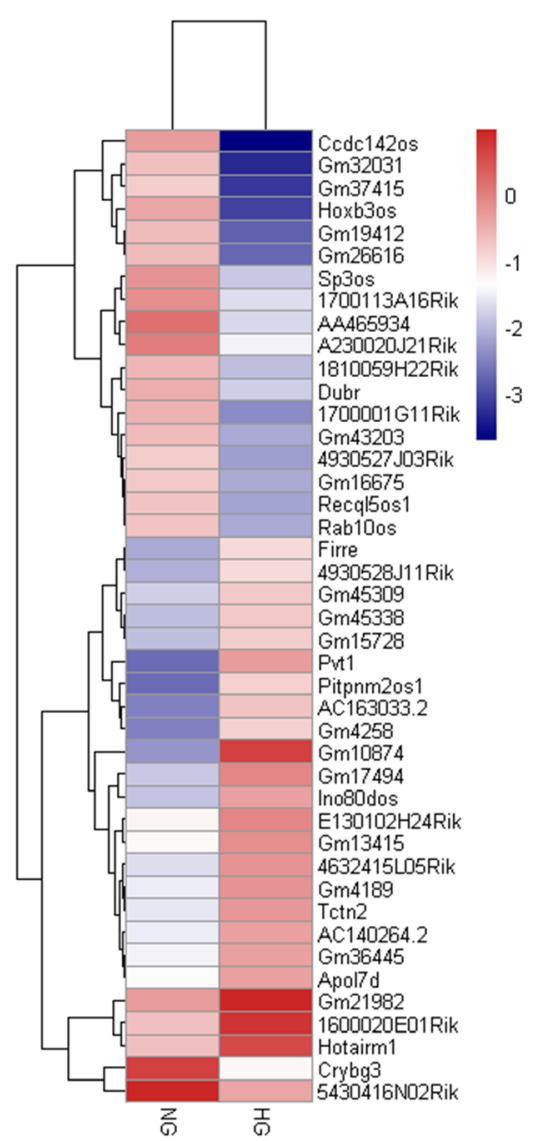

B

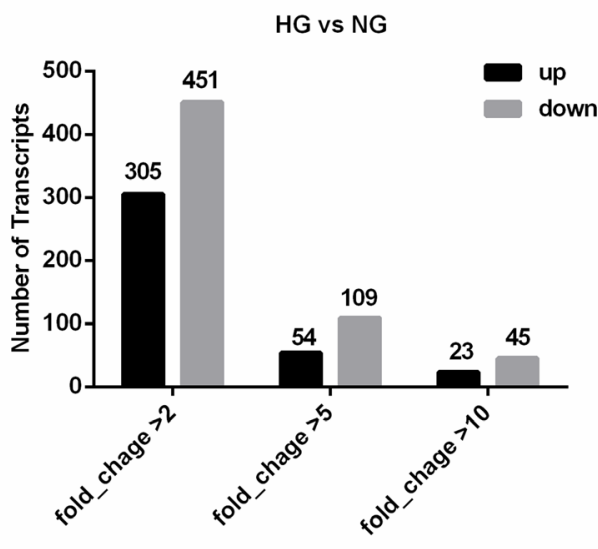

C

Figure 1. Hoxb3os was down-regulated in MPC5 cells treated with HG.

(A) Transcriptome sequencing was carried out. The heatmap showed the most significantly dysregulated IncRNAs. The lettering on the right indicates the corresponding specific gene names. The red color indicates "upregulation" and the blue color indicates "downregulation ". Color depth represents the degree of gene differential expression. (B) The dysregulated IncRNAs based on different fold changes.

(C) The upregulated and downregulated IncRNAs with the highest fold change. 
A

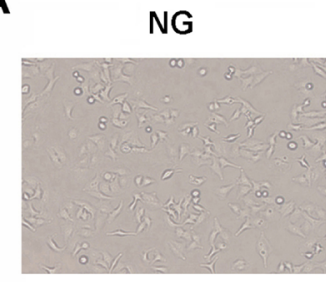

C

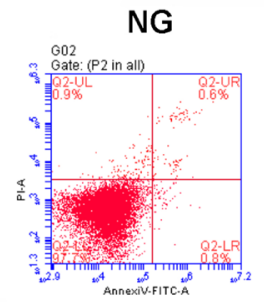

D

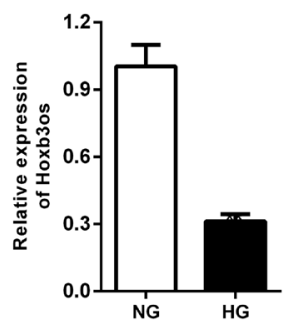

HG

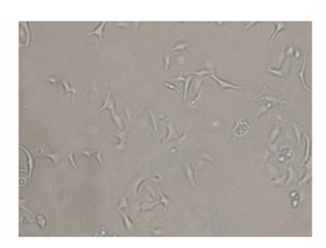

B

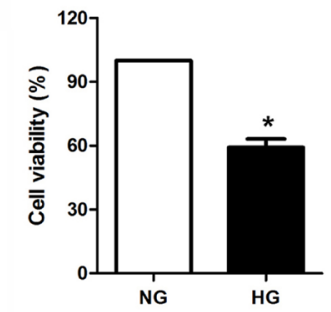

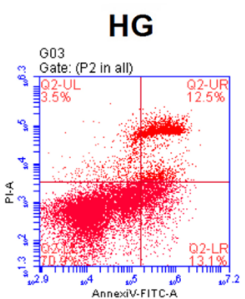

E
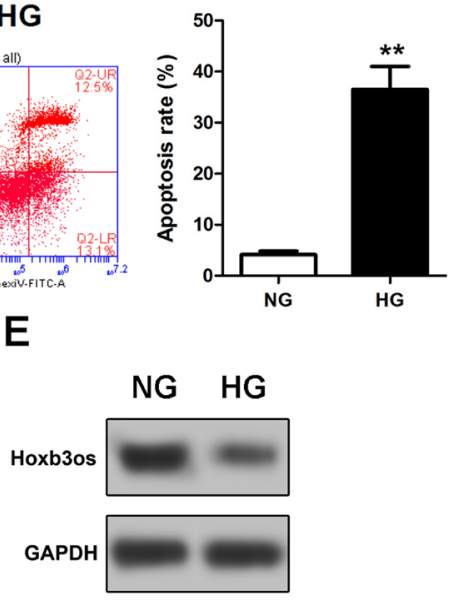

Figure 2. High-glucose treatment decreased the viability and induced apoptosis of MPC5 cells, and also inhibited the expression of Hoxb3os.

(A) The morphological changes of MPC5 cells under HG treatment. (B) CCK-8 assay was used to evaluate the viability of MPC5 cells after high-glucose treatment. (C) Flow cytometry was carried out to detect apoptosis of MPC5 cells. (D) qRT-PCR and (E) Northern blot were carried out to evaluate the expression of Hoxb3os in normal glucose- or high glucose-exposed MPC5 cells. The results were presented as the mean \pm S.D., $\mathrm{n}=3$, *vs NG $P<0.05,{ }^{* *}$ vs NG $P<0.01$.

\section{High glucose decreases the expression of Hoxb3os in podocytes}

In order to obtain podocyte injury model, MPC5 cells were challenged with high glucose to mimic the pathology condition during DN pathogenesis. High-glucose treatment led to prominent morphological changes in podocytes, as evidenced by their spindle shape compared to the typical epithelial-like morphology of non-treated cells (Fig. 2A). In addition, high-glucose challenge reduced podocytes viability and triggered their apoptosis (Fig. 2B-C), indicating the successful establishment of high glucose-induced podocyte damage model in vitro. Next, we evaluated the effect of high glucose on lncRNA Hoxb3os expression in podocytes. Consistently with our RNA-Seq data, qRT-PCR and Northern blotting results showed that the level of lncRNA Hoxb3os was significantly downregulated by high-glucose treatment (Fig. 2D-E).

\section{Hoxb3os is downregulated in the DN model}

Next, the in vivo study was employed to further confirm whether Hoxb3os is decreased in kidney tissue from DN mice. Compared to control $\mathrm{db} / \mathrm{m}$ mice, $\mathrm{db} / \mathrm{db}$ mice exhibited typical DN symptoms including markedly increased TUNEL-positive apoptotic cells in glomeruli (Fig. 3A), significantly higher levels of creatinine and urea nitrogen in the serum, and upregulated $24 \mathrm{~h}$ urinary protein concentration (Fig. 3B-D). These symptoms were indicative of kidney damage and proteinuria in $\mathrm{db} /$ $\mathrm{db}$ mice, confirming the successful establishment of the DN model. Then, we evaluated the expression level of Hoxb3os in the kidney tissue. In accordance with the in vitro study, it was notably downregulated in $\mathrm{db} / \mathrm{db}$ mice compared to $\mathrm{db} / \mathrm{m}$ mice (Fig. 3E).

\section{Overexpression of Hoxb3os reduces podocyte apoptosis induced by high glucose}

We next evaluated the cell viability and apoptosis of podocytes infected with lentivirus overexpressing Hoxb3os. The results revealed that the infection of exogenous Hoxb3os notably increased the expression of Hoxb3os in MPC5 cells (Fig. 4A). Overexpression of Hoxb3os reversed HG-induced decline in theviability of MPC5 cells (Fig. 4B). Moreover, Hoxb3os reduced the apoptosis of MPC5 cells induced by high-glucose treatment (Fig. 4C).

\section{Hoxb3os regulates autophagy level via Akt-mTOR signaling pathway in podocytes in the presence of high glucose}

Autophagy has profound effects on modulating podocyte functions. In order to ascertain whether Hoxb3os regulates high-glucose-induced podocytes damage in an autophagy-dependent manner, we performed Western blot assay to detect the expression of relevant proteins involved in autophagy. The results indicated that highglucose treatment inhibited the ratio of LC3II/I and increased the expression of $\mathrm{p} 62$, while Hoxb3os overexpression suppressed the effect of high glucose on the ratio of LC3II/I and p62 in MPC5 cells (Fig. 5), suggesting that Hoxb3os reversed high glucose-induced autophagy inhibition. Subsequently, we investigated the effect of lncRNA Hoxb3os on Akt-mTOR signaling, a predominant autophagy-modulating pathway. The results showed that 
A

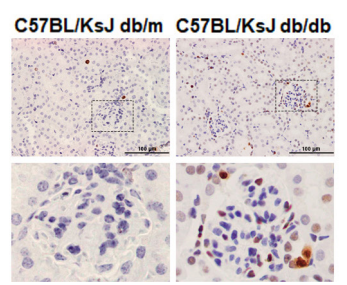

C

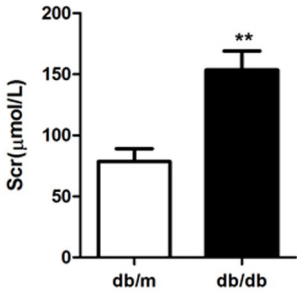

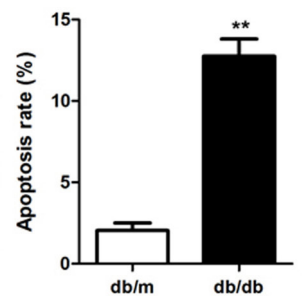

B

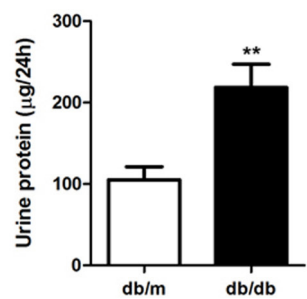

D

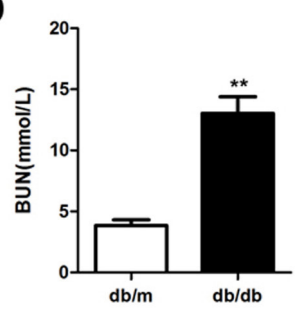

E

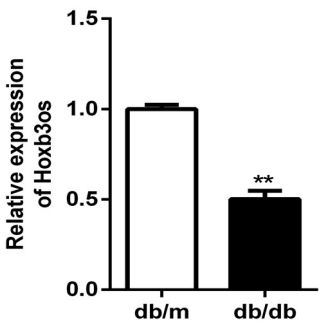

Figure 3. Hoxb3os was downregulated in DN mice.

(A) TUNEL assay was performed to detect the apoptosis in kidney cortex tissue. The lower images represent the zoomed area or TUNELpositive cells. (B) The protein concentration in the urine of mice was evaluated using BCA kit. (C-D) The level of Scr and BUN in the serum of mice were evaluated using ELISA. (E) qRT-PCR was carried out to evaluate the expression of Hoxb3os. The results were presented as the mean \pm S.D., $\mathrm{n}=6,{ }^{* *} v s \mathrm{db} / \mathrm{m}$ group, $P<0.01$.

A

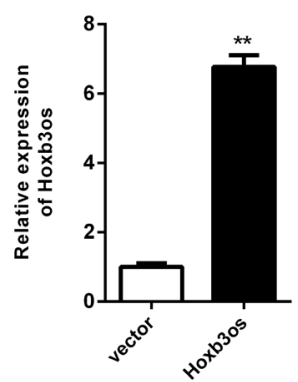

C

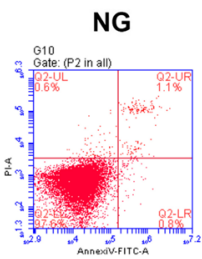

HG+vector

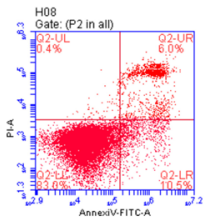

B

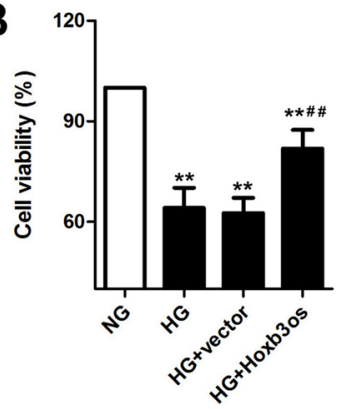

HG

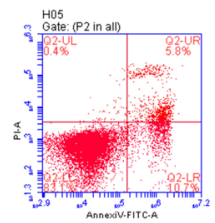

HG+Hoxb3os
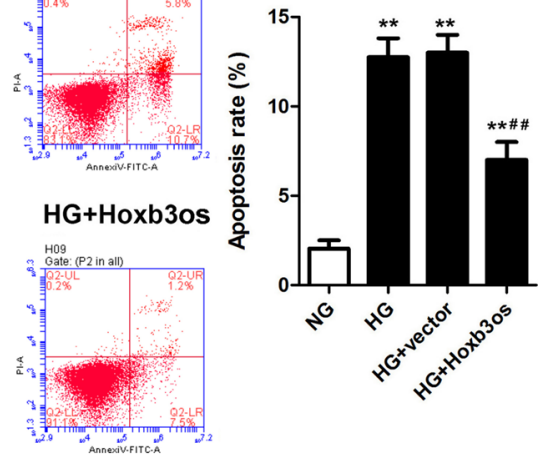

Figure 4. Hoxb3os overexpression protected MPC5 cells from HG-induced cell injury.

(A) qRT-PCR was used to evaluate the expression of Hoxb3os. (B) CCK-8 assay was used to evaluate the viability of MPC5 cells after highglucose treatment and lentivirus infection. (C) Flow cytometry was carried out to detect the apoptosis of MPC5 cells. The results were presented as the mean \pm S.D., $\mathrm{n}=3,{ }^{* *} v s \mathrm{NG}$ or vector $P<0.01$, \#\#VS HG+vector $P<0.01$.

high-glucose treatment markedly induced the phosphorylation of mTOR and Akt, which was inhibited by lncRNA Hoxb3os overexpression (Fig. 5), suggesting that Hoxb3os possibly reversed high glucose-induced autophagy inhibition in an Akt-mTOR signaling-dependent manner. Based on the negative association between Sirtuin 1 (SIRT1) and mTOR signaling pathway, we further assessed SIRT1 expression in normal/high glucose-exposed MPC5 cells in the presence/absence of exogenous Hoxb3os. The results showed that HG exposure significantly decreased mRNA and protein levels of SIRT1 in MPC5 cells, while Hoxb3os overexpression reversed the decline in SIRT1 expression in HG-exposed MPC5 cells (Fig. S1A-B at https:// ojs.ptbioch.edu.pl/index.php/abp/). By contrast, administration of transcription inhibitor ACTD or translational inhibitor CHX decreased the protein level of SIRT1 in HG-treated cells, whereas Hoxb3os overexpression significantly increased the expression of SIRT1 in MPC5 cells treated with ACTD, but did not affect SIRT1 expression in those cells upon treatment with CHX (Fig. S1C at htt- 

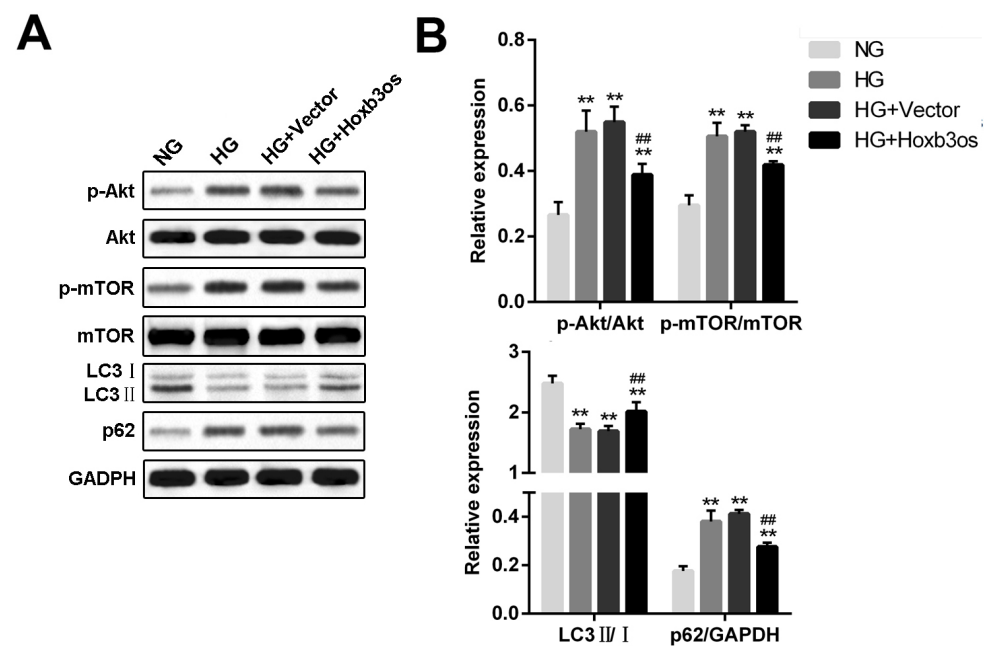

Figure 5. Hoxb3os regulated Akt-mTOR signaling.

(A-B) Western blot was used to investigate the autophagy level and the activation of Akt-mTOR signaling pathway in MPC5 cells treated with lentivirus in the presence of NG or HG. The results were presented as the mean \pm S.D., $\mathrm{n}=3$, **vs NG or vector $P<0.01$, \#\#vs HG+vector $P<0.01$.

ps://ojs.ptbioch.edu.pl/index.php/abp/), which implied that Hoxb3os did not affect the gene transcription nor protein degradation process. Hoxb3os-mediated increased SIRT1 level might be related to the post-transcriptional regulatory mechanisms, such as the translation process and mRNA stability. Taken together, lncRNA Hoxb3os prevented high glucose-induced autophagy inhibition by restraining Akt-mTOR pathway activation in podocytes, and the process might be SIRT1-dependent.

\section{DISCUSSION}

It is well known that podocytes play a crucial role in the development of DN. They are specialized glomerular epithelial cells that form a layer of the filtration barrier in the kidney and they are terminally differentiated with limited capacity to renew. In animal models, podocyte injury and loss occur early during the course of DN (Susztak et al., 2004). Podocytes foot processes are interconnected by slit diaphragms and form the final filtration barrier. It was reported that the reduction in podocytes number mediated by apoptosis is observed in patients with both early and late DN as well as in the DN animal models. Increasing evidence has indicated that podocyte apoptosis coincides with albuminuria onset and precedes podocytopenia in different mouse models of diabetes. Therefore, clarifying the modulatory mechanisms underlying high glucose-triggered podocyte damage is thought to be critical for understanding the pathogenesis of diabetic kidney disease (Reidy et al., 2014) and is of great importance for the development of the prognostic and therapeutic targets in DN.

LncRNAs were reported to play crucial roles in regulating high glucose-triggered podocyte damage. For example, the level of MALAT1 is significantly increased in the renal cortex from STZ-challenged DN mice, as well as in high glucose-stimulated podocytes (Reidy et al., 2014). The upregulated MALAT1 exerts a detrimental effect on podocytes by modulating $\beta$-catenin signaling (Reidy et al., 2014). LncRNA SOX2OT alleviates high glucose-induced podocyte damage via regulating miR-9/SIRT1 axis-mediated cell autophagy (Zhang et al., 2019). LncRNA LINK-A inhibits the apoptosis of DN podocytes by activating $\mathrm{HIF}-1 \alpha$ signaling (Yang et al., 2019). The expression of lncRNA PVT1 is high in DN patients, and it is further upregulated after highglucose treatment (D. W. Liu et al., 2019). LncRNA PVT1 inhibition prevents podocyte apoptosis by activating FOXA1 (Liu et al., 2019a). In the present study, we found multiple differentially-expressed lncRNAs in high glucose-treated podocytes. Among the top 5 increased and top 5 decreased lncRNAs, there was an identified lncRNA PVT1, as well as several novel lncRNAs such as Gm10874, Gm17494, AC163033.2, Pitpnm2os1, Ccdc142os, Gm32031, Gm37415 and Gm19412. LncRNA Hoxb3os was the second most downregulated by highglucose challenge. It was reported that Hoxb3os is a kidney-specific lncRNA mainly expressed in kidney tubules (Aboudehen et al., 2018). Compared to the healthy mice, Hoxb3os expression was reduced in the hydatoncus epithelium in $\mathrm{Pkd} 1$ and $\mathrm{Pkd} 2$ mutated mice with polycystic kidney disease (Aboudehen et al., 2018). The expression of lncRNA HOXB-AS1, a cognate of Hoxb3os, was also reduced in the renal tissues of polycystic kidney disease patients (Aboudehen et al., 2018). To date, the regulatory roles of lncRNA Hoxb3os and its cognate lncRNAs in DN and high glucose-induced podocyte damage are largely unknown. In our present work, we reported for the first time that lncRNA Hoxb3os could serve as a protective lncRNA in DN podocyte damage, and the underlying mechanisms. We found significantly downregulated lncRNA Hoxb3os expression in the renal tissues from $\mathrm{db} / \mathrm{db} \mathrm{DN}$ mice, as well as in high glucose-treated MPC5 cells. The functional study revealed that Hoxb3os was capable of reversing the viability decrease and apoptosis promotion caused by HG treatment in MPC5 cells. These findings suggested the critical role of Hoxb3os in the development of DN.

Autophagy plays pivotal roles in maintaining cell functions by supporting the metabolic needs and the renewal of certain organelles. Impaired autophagy is also closely related to the disrupted podocyte functions and DN progression (Tagawa et al., 2016). Activation of autophagy by berberine and sclerodermatin was reported to alleviate high glucose-induced podocyte damage (Jin et al., 2017; Wu et al., 2018). Our previous works also found that activating cell autophagy was an effective way to prevent podocyte damage and DN development (Jin et al., 2019a; Jin et al., 2019b). LncRNAs are important regulators of autophagy- 
related pathways. SPAG5-AS1 aggravates high glucoseinduced podocyte damage by activating mTOR signaling and autophagy inhibition (Xu et al., 2020). Importantly, lncRNA Hoxb3os deficiency was reported to promote mTOR phosphorylation (Brosius \& He, 2015). Therefore, we investigated the effect of exogenous lncRNA Hoxb3os on mTOR signaling and podocyte autophagy after highglucose treatment and found that high glucose-induced autophagy inhibition and Akt-mTOR signaling activation in MPC5 cells could be reversed by lncRNA Hoxb3os overexpression, indicating its crucial role in autophagymediated podocyte function maintenance. Accumulating evidence has suggested that SIRT1 can negatively regulate mTOR signaling pathway (Chen et al., 2019a). In addition to that, our finding further confirmed that lncRNA Hoxb3os-induced SIRT1 level increase might result in the inhibition of mTOR phosphorylation.

LncRNAs regulate cell functions typically through their "molecular sponge" role by competitive binding to miRNAs, thus antagonizing the negative effect of miRNAs on their target mRNAs. For example, lncRNA SOX2OT increases SIRT1 expression by binding to miR-9, thus alleviating high glucose-induced podocyte damage (Zhang et al., 2019). LncRNA HOXB-AS1, the human counterpart of lncRNA Hoxb3os, promotes the growth and migration of glioblastoma by counteracting the inhibitory effect of miR-885-3p on HOXB2 expression (Chen et al., 2019b). In addition, lncRNA HOXB-AS1 was reported to interact with RNA-binding protein (RBP) ELAVL1 and alter its capacity to bind to the FUT4 mRNA, thus affecting the stability of FUT4 mRNA and regulating multiple myeloma growth (Chen et al., 2020). To establish whether LncRNA Hoxb3os modulates Akt-mTOR signaling, SIRT1 expression and autophagy through ceRNA function or RBPbinding capacity further exploration in our future studies is still needed.

In conclusion, our research revealed that the novel lncRNA Hoxb3os played a protective effect on HGinduced podocyte injury. The mechanism was involved in the regulation of the autophagy depending on AktmTOR signaling pathway. We speculate that targeting Hoxb3os may be a promising therapeutic approach for the prevention and treatment of DN. However, the further molecular mechanism through which Hoxb3os modulates the Akt-mTOR signaling pathway needs to be elucidated in the future.

\section{REFERENCES}

Aboudehen K, Farahani S, Kanchwala M, Chan SC, Avdulov S, Mickelson A, Lee D, Gearhart MD, Patel V, Xing C, Igarashi P (2018) Long noncoding RNA Hoxb3os is dysregulated in autosomal dominant polycystic kidney disease and regulates mTOR signaling. $J$ Biol Chem 293: 9388-9398. https://doi.org/10.1074/ibc.RA118.001723

Bai X, Geng J, Li X, Wan J, Liu J, Zhou Z, Liu X (2018) Long noncoding RNA LINC01619 regulates microRNA-27a/forkhead box protein 01 and endoplasmic reticulum stress-mediated podocyte injury in diabetic nephropathy. Antioxid Redox Signal 29: 355-376. https://doi.org/10.1089/ars.2017.7278

Brosius FC, 3rd, He JC (2015) JAK inhibition and progressive kidney disease. Curr Opin Nephrol Hypertens 24: 88-95. https://doi. org/10.1097/MNH.0000000000000079

Chen P, Chen F, Lei J, Li Q, Zhou B (2019a) Activation of the miR-34a-mediated SIRT1/mTOR signaling pathway by urolithin a attenuates D-galactose-induced brain aging in mice. Neurotherapeutics 16: 1269-1282. https://doi.org/10.1007/s13311-019-00753-0

Chen X, Li LQ, Qiu X, Wu H (2019b) Long non-coding RNA HOXB-AS1 promotes proliferation, migration and invasion of glioblastoma cells via HOXB-AS1/miR-885-3p/HOXB2 axis. Neoplasma 66: 386-396. https://doi.org/10.4149/neo_2018_180606N377

Chen R, Zhang X, Wang C (2020) LncRNA HOXB-AS1 promotes cell growth in multiple myeloma via FUT4 mRNA stability by ELAVL1. J Cell Biochem 121: 4043-4051. https://doi.org/10.1002/jcb.29573
Emmrich S, Streltsov A, Schmidt F, Thangapandi VR, Reinhardt D, Klusmann JH (2014) LincRNAs MONC and MIR100HG act as oncogenes in acute megakaryoblastic leukemia. Mol Cancer 13: 171. https://doi.org/10.1186/1476-4598-13-171

Gaede P, Lund-Andersen H, Parving HH, Pedersen O (2008) Effect of a multifactorial intervention on mortality in type 2 diabetes. $N$ Engl J Med 358: 580-591. https://doi.org/10.1056/NEJMoa0706245

Haneda M, Utsunomiya K, Koya D, Babazono T, Moriya T, Makino H, Kimura K, Suzuki Y, Wada T, Ogawa S, Inaba M, Kanno Y, Shigematsu T, Masakane I, Tsuchiya K, Honda K, Ichikawa K, Shide K (2015) A new classification of diabetic nephropathy 2014: a report from Joint Committee on Diabetic Nephropathy. Clin Exp Nephrol 19: 1-5. https://doi.org/10.1007/s10157-014-1057-z

Jin Y, Liu S, Ma Q, Xiao D, Chen L (2017) Berberine enhances the AMPK activation and autophagy and mitigates high glucose-induced apoptosis of mouse podocytes. Eur J Pharmacol 794: 106-114. https://doi.org/10.1016/j.ejphar.2016.11.037

Jin J, Gong J, Zhao L, Zhang H, He Q, Jiang X (2019a) Inhibition of high mobility group box 1 (HMGB1) attenuates podocyte apoptosis and epithelial-mesenchymal transition by regulating autophagy flux. J Diabetes 11: 826-836. https://doi.org/10.1111/1753-0407.12914

Jin J, Shi Y, Gong J, Zhao L, Li Y, He Q, Huang H (2019b) Exosome secreted from adipose-derived stem cells attenuates diabetic nephropathy by promoting autophagy flux and inhibiting apoptosis in podocyte. Stem Cell Res Ther 10: 95. https://doi.org/10.1186/ s13287-019-1177-1

Li A, Peng R, Sun Y, Liu H, Peng H, Zhang Z (2018) LincRNA 1700020I14Rik alleviates cell proliferation and fibrosis in diabetic nephropathy via miR-34a-5p/Sirt1/HIF-1alpha signaling. Cell Death Dis 9: 461. https://doi.org/10.1038/s41419-018-0527-8

Li SY, Susztak K (2016) The long noncoding RNA Tug1 connects metabolic changes with kidney disease in podocytes. I Clin Invest 126: 4072-4075. https://doi.org/10.1172/JCI90828

Liu DW, Zhang JH, Liu FX, Wang XT, Pan SK, Jiang DK, Zhao ZH, Liu ZS (2019a) Silencing of long noncoding RNA PVT1 inhibits podocyte damage and apoptosis in diabetic nephropathy by upregulating FOXA1. Exp Mol Med 51: 1-15. https://doi.org/10.1038/ s12276-019-0259-6

Liu J, Li QX, Wang XJ, Zhang C, Duan YQ, Wang ZY, Zhang Y, Yu X, Li NJ, Sun JP, Yi F (2016b) beta-Arrestins promote podocyte injury by inhibition of autophagy in diabetic nephropathy. Cell Death Dis 7: e2183. https://doi.org/10.1038/cddis.2016.89

Long J, Badal SS, Ye Z, Wang Y, Ayanga BA, Galvan DL, Green NH, Chang BH, Overbeek PA, Danesh FR (2016) Long noncoding RNA Tug1 regulates mitochondrial bioenergetics in diabetic nephropathy. J Clin Invest 126: 4205-4218. https:/ /doi.org/10.1172/ JCI87927

Marshall SM (2012) Diabetic nephropathy in type 1 diabetes: has the outlook improved since the 1980s? Diabetologia 55: 2301-2306. https://doi.org/10.1007/s00125-012-2606-1

Reidy K, Kang HM, Hostetter T, Susztak K (2014) Molecular mechanisms of diabetic kidney disease. J Clin Invest 124: 2333-2340. https://doi.org/10.1172/JCI72271

Rosolowsky ET, Skupien J, Smiles AM, Niewczas M, Roshan B, Stanton R, Eckfeldt JH, Warram JH, Krolewski AS (2011) Risk for ESRD in type 1 diabetes remains high despite renoprotection. I Am Soc Nephrol 22: 545-553. https://doi.org/10.1681/ASN.2010040354

Susztak K, Böttinger E, Novetsky A, Liang D, Zhu Y, Ciccone E, Wu D, Dunn S, McCue P, Sharma K (2004) Molecular profiling of diabetic mouse kidney reveals novel genes linked to glomerular disease. Diabetes 53: 784-794. https://doi.org/10.2337/diabetes.53.3.784

Tagawa A, Yasuda M, Kume S, Yamahara K, Nakazawa J, Chin-Kanasaki M, Araki H, Araki S, Koya D, Asanuma K, Kim EH, Haneda M, Kajiwara N, Hayashi K, Ohashi H, Ugi S, Maegawa H, Uzu T (2016) Impaired podocyte autophagy exacerbates proteinuria in diabetic nephropathy. Diabetes 65: 755-767. https:///doi.org/10.2337/ db15-0473

Wu F, Li S, Zhang N, Huang W, Li X, Wang M, Bai D, Han B (2018) Hispidulin alleviates high-glucose-induced podocyte injury by regulating protective autophagy. Biomed Pharmacother 104: 307-314. https://doi.org/10.1016/j.biopha.2018.05.017

Xu J, Deng Y, Wang Y, Sun X, Chen S, Fu G (2020) SPAG5-AS1 inhibited autophagy and aggravated apoptosis of podocytes via SPAG5/AKT/mTOR pathway. Cell Prolif 53: e12738. https://doi. org $/ 10.1111 / \mathrm{cpr} .12738$

Yang J, Li L, Hong S, Zhou Z, Fan W (2019) LINK-A lncRNA activates HIF1alpha signaling and inhibits podocyte cell apoptosis in diabetic nephropathy. Exp Ther Med 18: 119-124. https://doi. org/10.3892/etm.2019.7542

Zhang Y, Chang B, Zhang J, Wu X (2019) LncRNA SOX2OT alleviates the high glucose-induced podocytes injury through autophagy induction by the miR-9/SIRT1 axis. Exp Mol Pathol 110: 104283. https://doi.org/10.1016/j.yexmp.2019.104283 